DOI: $10.17516 / 1997-1397-2021-14-1-42-46$

УДК $532.527+532.517 .4$

\title{
Mass Transfer Between the Vortex Ring and the Surrounding Fluid, when the Density of the Fluid in the Vortex is Less than Outside it
}

\author{
Victor V. Nikulin* \\ Eugene A. Chashnikov ${ }^{\dagger}$ \\ Institute of Hydrodynamics M. A. Lavrentieva SB RAS \\ Novosibirsk, Russian Federation
}

Received 11.06.2020, received in revised form 24.08.2020, accepted 12.10.2020

\begin{abstract}
The mass transfer between the atmosphere of a vortex ring and the surrounding liquid was studied by the shadow method in the case, when the density of the liquid in the vortex is less than outside it. The obtained results were compared with experiments on the motion of vortex rings containing a fluid denser, than the surrounding fluid. The qualitative effects of changing the shadow image is established to be the same in both cases. The characteristic path and time of exchange are determined by analyzing of shadow images in depending on the speed and magnitude of the density difference.
\end{abstract}

Keywords: turbulent vortex ring, mass transfer.

Citation: V.V. Nikulin, E.A. Chashnikov, Mass Transfer Between the Vortex Ring and the Surrounding Fluid, when the Density of the Fluid in the Vortex is Less than Outside it, J. Sib. Fed. Univ. Math. Phys., 2021, 14(1), 42-46. DOI: 10.17516/1997-1397-2021-14-1-42-46.

A vortex ring is a toroidal volume of a swirling liquid or gas (the core of the vortex), and some volume of fluid called the atmosphere of the vortex [1] or bubble [2], which moves with the core. Between the atmosphere of the vortex and the surrounding fluid there is a continual mass transfer. The environment is drawn into the atmosphere, mixed with the liquid there, and almost the same amount of water goes out of it. At the same time, slightly more liquid remains inside than enters it, which provides to a slow increase in the volume of the atmosphere [2].

The study of mass transfer between the atmosphere of a vortex ring and the surrounding fluid is of fundamental and applied value. Scientific significance is due to the fundamental nature of the problem of mass transfer in hydrodynamics and the fact that the vortex ring is an example of an isolated concentrated vortex, therefore, the laws established during its study are associated with the vortex nature of the flow and can be transferred to other concentrated vortices. The practical significance is associated with the fact that vortex rings often arise in various technogenic and natural processes (for example, during explosions, volcanic eruptions). The transfer of fine powder by vortex rings has found application in the vortex-powder method of extinguishing fires in emergency gushing gas and oil wells [1]. In these cases, it is necessary to be able to evaluate the characteristics of turbulent exchange, since as a result of it the entrainment of impurities, heat, and vorticity from the vortex takes place.

The study of mass transfer is significantly complicated by the unsteadiness of the flow, since the vortex cannot be stopped in space. Therefore, studies were limited to observing the loss

\footnotetext{
*nikulin@hydro.nsc.ru https://ORCID 0000-0001-8996-6490

†chashnikov.e.a@gmail.com https://ORCID 0000-0002-1775-1703

(C) Siberian Federal University. All rights reserved
} 
of passive impurity. The loss of a passive impurity by a vortex ring due to mass transfer was experimentally studied in [3]. It turned out that the impurity is lost quickly, about half of it after passing about 4 diameters of the ring. An empirical dependence of the fraction of the transferred impurity on the path traveled by the vortex ring is obtained. However, the characteristic distance at which the turbulent exchange takes place, and its dependence on the motion parameters, have not been established.

A new method of observing mass transfer between a vortex ring and the environment was proposed in $[4,5]$. The density of the liquid in vortex rings differs from the density of the liquid in the environment; density is greater [4] or less [5]. A vortex is observed in the process of movement by the direct shadow method. Its visualization is due to optical inhomogeneities occurring during mass transfer by liquids with different optical densities inside and outside the vortex. In this case, the shadow image first darkens, reaches a maximum of darkening, then brightens. Because this effect is associated with mass transfer, the presence of a maximum of darkening allows us to introduce the characteristic spatial and temporal scales of the exchange. Observation of the loss of the passive impurity does not allow the introduction of such a scale. The image brightens continuously, so it is impossible to establish any vortex position or exchange time, which stands out among others and can be taken as characteristic. This is the advantage of this visualization method.

In order to make sure that the observed effect is not caused by the difference in densities, but due to the hydrodynamics of the flow, experiments with a different sign of the difference in the densities of the liquids in the vortex and outside it are necessary. The case with the greater density of the fluid in the vortex than outside it is considered in sufficient detail in [4]. At the same time, the results for the opposite density difference, presented in [5], are preliminary, concern only one value of the density difference and contain a small number of experimental points. In the present work, the number of experimental points is increased and two values of the density difference are considered, which, as will be shown, are sufficient. The obtained results are compared with experiments on the motion of vortex rings containing a fluid denser than the surrounding fluid. An explanation of the effect of changing the shadow image is proposed.

\section{The experimental results}

The experimental setup is similar to [5]. It is a vertical vessel $300 \mathrm{~mm}$ high, with a cross section of $150 \times 150 \mathrm{~mm}$, filled with a two-layer liquid: the upper layer is water with a density $\rho_{1}=1 \mathrm{~g} / \mathrm{cm}^{3}$, depth $100 \mathrm{~mm}$, the lower layer is a sugar solution of various densities $\rho_{2}=1.08$, $1.04 \mathrm{~g} / \mathrm{cm}^{3}$ and $150 \mathrm{~mm}$ deep. Vortex rings are formed in the lower layer by pushing a pulse jet of $28 \mathrm{~mm}$ length through a nozzle with a diameter of $12.5 \mathrm{~mm}$. The distance from the nozzle exit to the interface is $50 \mathrm{~mm}$. A vortex is formed in the upper layer and goes into the lower one, remaining filled with the liquid less dense than the surrounding one. The motion of the vortex ring is recorded by a high-speed video camera. Shadow is shot in the perpendicular direction of movement with a frequency of 125 to 1000 frames per second. As the parameters of the vortex, its velocity $u$ and the radius of the axial line of the toroidal core $R$ at the moment when the vortex is at a distance of $20-25 \mathrm{~mm}$ from the interface are taken. Based on these values and the kinematic viscosity of water, taken equal to $10^{-2} \mathrm{~cm}^{2} / \mathrm{s}$, the Reynolds number is calculated. The average diameter of the vortex ring and its dispersion are $(14.6 \pm 0.6) \mathrm{mm}$ in all experiments. The diffusion coefficient of a sugar solution in water is $3 * 10^{-6} \mathrm{~cm}^{2} / \mathrm{s}$. Travel time less than $0.5 \mathrm{~s}$. Then the characteristic path of molecular diffusion is less than $1.2 * 10^{-3} \mathrm{~cm}$, which is 
much smaller than the size of the vortex. Therefore, molecular diffusion can be neglected.

Fig. 1 shows typical shadow photographs of a vortex at various distances from the interface. They demonstrate the observed effect: first, the atmosphere is transparent, mainly its border is visible, Fig. 1a, then the image darkens, reaches a maximum of darkening, then brightens.

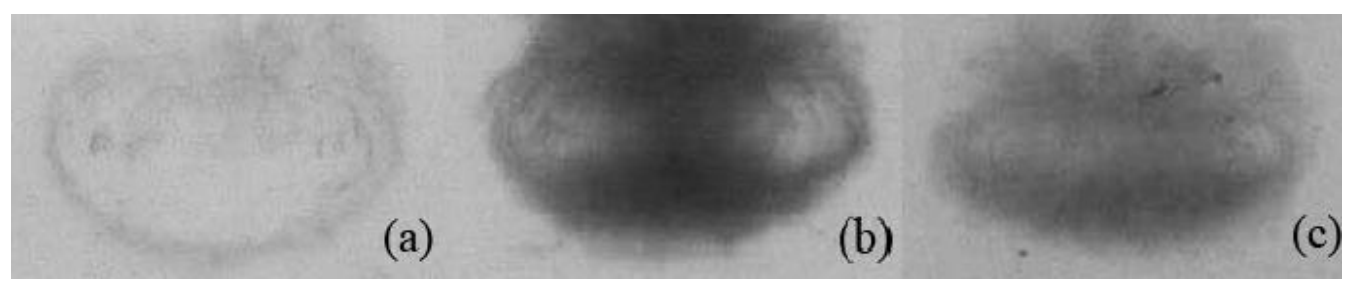

Fig. 1. Shadow photographs of a vortex ring at various distances from the interface: 18 (a), 40 (b) and $120 \mathrm{~mm}(\mathrm{c})$. Vortex velocity $\mathrm{u}=2.7 \mathrm{~m} / \mathrm{s}, \mathrm{Re}=19300$

The effect is due to the fact that due to mass transfer, the homogeneity of the media is violated, the external liquid penetrates into the atmosphere, and the internal goes out, resulting in density gradients and shadow visualization. For a vortex ring it looks like a sharp darkening of its atmosphere. First, the darkening increases, Fig. 1b and then, starting from a certain distance, decreases, Fig. 1c. This means that the original fluid is replaced by the surrounding. In this case, the core of the vortex remains lighter, i.e. there is practically no mass transfer with the core. The value of the average blackening per pixel of the region corresponding to the atmosphere of the vortex ring is found from shadow images. This region is determined immediately after the exit of the vortex from the lower layer, when it is clearly visible, and is considered unchanged throughout the entire observation path. The initial velocity of the vortex in experiments is varied. The distance $z$ from the interface to the vortex ring is the distance from the boundary to the center line of the toroidal nucleus, since the nucleus is parallel to the boundary. The average velocity in distance by $10,20 \mathrm{~mm}$ is accepted as the instant velocity. The graph of the dependence of blackening on the distance to the interface in $[4,5]$ is always shown to have a distinct maximum, that is reached at a certain distance $z^{*}$. This distance is taken as a characteristic spatial scale of mass transfer, since darkening is associated with mass transfer. The maximum darkening is assumed to be achieved with maximum mixing, i.e. approximately half of the original fluid is replaced by the surrounding. Along with the spatial scale, a characteristic time scale of mass transfer is introduced, which is equal to: $t^{*}=z^{*} / u$. Therefore, there are an ability to obtain the dimensionless spatial and temporal scale $\tau=z^{*} / R=t^{*} * u / R$ by using the radius of the ring $R$ as a dimensionless spatial scale, and $R / u$ as a temporary one. The vortex ring velocity in the experiments varied in the range from 30 to $470 \mathrm{~cm} / \mathrm{s}$, which corresponded to a change in the Reynolds number from 2300 to 35000. Fig. 2 shows the dependence of $\tau$ on the Reynolds number for two values $\rho_{2}=1.04,1.08 \mathrm{~g} / \mathrm{cm}^{3}$. The shadow imaging does not occur, when the density of the solution is less than $1.04 \mathrm{~g} / \mathrm{cm}^{3}$. However, as follows from Fig. 2, the results for densities of 1.04 and $1.08 \mathrm{~g} / \mathrm{cm}^{3}$ are close, therefore, a further decrease in $\rho_{2}$ is not required.

\section{Analysis of the results}

As follows from Fig. 2, the quantity $\tau$ decreases with increasing Reynolds number, and at $10^{4}$, $\tau$ is approximately constant, and we can put $\tau=\tau^{*} \approx$ const. Its average value and dispersion 


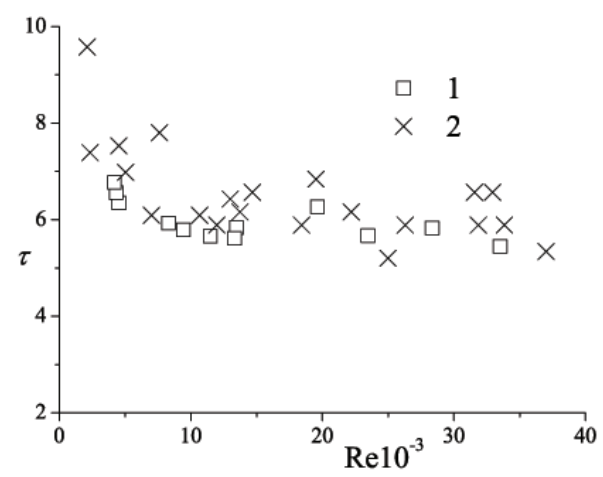

Fig. 2. The dimensionless characteristic scale of mass transfer depending on the Reynolds number of the vortex at various densities of the lower layer: $\rho_{2}=1.04(1)$ and $1.08 \mathrm{~g} / \mathrm{cm}^{3}(2)$

at $\operatorname{Re}>10^{4}$ are equal: $\tau^{*}=6 \pm 0.5$. This means that the characteristic path and mass transfer time decrease with velocity increase, and for large $R e$ the path is approximately constant, and time is inversely proportional to speed.

The changes in shadow images for ouer case and for vortex rings containing a fluid denser than the surrounding one is shown to be similar character. According to [4], at $\operatorname{Re}>10^{4}$, the characteristic exchange path is practically independent of the velocity and is approximately equal to $4 R$. In the present work, for $\operatorname{Re}>10^{4}$, the path is also practically independent of speed and is equal to $6 R$. Thus, the qualitative effects are similar, but there are quantitative differences. From this we can conclude that the effect of changing the shadow image is due to the hydrodynamics of the flow and is not caused by the difference in densities. However, the difference in density has an effect on it.

The observed effect can be explained as follows. We believe that the main mechanism of mass transfer between the atmosphere of a turbulent vortex ring and the environment is turbulent diffusion. As a result, according to the Prandtl approach, liquid volumes from the outside penetrate into the atmosphere, and from the inside out. Inside the atmosphere, due to rotation around the nucleus, liquid volumes received from outside are distributed throughout the atmosphere. Since the density of liquids inside and out, and therefore the refractive indices are different, sharp gradients of the refractive index appear on these liquid volumes, leading to shadow imaging. Initially, with an increase in their number, darkening occurs. Then, when the outer fluid replaces more than half of the inner fluid, the shadow image begins to brighten. For this reason, qualitative effects are independent of the sign of the density difference. However, the quantitative effects are different. This is due to the fact that the mass transfer time depends on the value of the coefficient of turbulent diffusion in the atmosphere of the vortex ring. The fact that the exchange time in the case when the fluid density in the vortex is shorter than the outside exceeds the exchange time for the opposite density difference, means that the coefficient of turbulent diffusion at a lower density will be less.

\section{Conclusion}

The shadow method considers the motion of a vortex ring, initially filled with a liquid of lower density than the surrounding one. The qualitative change in the shadow image has been 
established to be completely analogous to the case when the vortex is initially filled with a fluid more dense than the surrounding one: the image first darkens, the darkening reaches a maximum, then brightens. Thus, the observed effect is caused by the hydrodynamics of the flow, and not by the difference in densities. The presence of a maximum of darkening allows us to introduce the characteristic spatial and temporal scales of mass transfer. The effect is explained by the fact that the mass transfer between the atmosphere of the vortex ring and the surrounding liquid is mainly due to turbulent diffusion. In this case, the quantitative differences in the spatial and temporal scales of mass transfer in the case of opposite differences in the density of the liquid in the vortex and outside it are explained by the difference in the turbulent diffusion coefficients. The core remains to be lighter by the suppression of turbulence in the core of the vortex ring [6].

This work was financially supported by the RFBR, grant no. 18-08-00824.

\title{
References
}

[1] D.G.Akhmetov, Vortex Rings, Springer, Berlin, 2009.

[2] T.Maxworthy, Turbulent vortex rings, J. Fluid. Mech., 64(1974), 227-239.

[3] V.F.Tarasov, V.I.Yakushev, Transport in a turbulent vortex ring, J. Appl. Mech. Tech. Phys., 15(1974), 106-112.

[4] V.V.Nikulin, Shadowgraph visualization of turbulent exchange between a vortex ring and the environment at different densities of the fluid in the vortex interior and exterior, Technical Physics Letters, 45(2019), no. 4, 314-316. DOI: 10.1134/S1063785019040114

[5] V.V.Nikulin, R.A.Panenko, Experimental observation of turbulent exchange between heterogeneous vortex ring and surrounding medium by shadowgraph method, J. Phys.: Conf. Ser., 1421(2019), 012006.

[6] V.Nikulin, S.Savtchenko, N.Ashgriz, A model for the turbulent suppression in swirling flows, Phys. Letters. A, 381(2017), 3989-3995. DOI: 10.1016/j.physleta.2017.10.028

\section{Массообмен между вихревым кольцом и окружающей средой в случае, когда Плотность жидкости в вихре меньше, чем вне его}

\section{Виктор В. Никулин \\ Евгений А. Чашников}

Институт гидродинамики им. М. А. Лаврентьева СО РАН

Новосибирск, Российская Федерация

\begin{abstract}
Аннотация. Теневым методом исследован массообмен между атмосферой вихревого кольца и окружающей жидкостью в случае, когда плотность жидкости в вихре меньше, чем вне его. Проведено сравнение полученных результатов с экспериментами по движению вихревых колец, содержащих жидкость более плотную, чем окружающая. Установлено, что качественные эффекты изменения теневого изображения в обоих случаях одинаковы. Путем анализа теневых изображений определены характерные путь и время обмена в зависимости от скорости и величины разности плотностей.
\end{abstract}

Ключевые слова: турбулентное вихревое кольцо, массообмен. 